\title{
Placebo Administration
}

National Cancer Institute

\section{Source}

National Cancer Institute. Placebo Administration. NCI Thesaurus. Code C116558.

The use of a medically inactive substance or intervention in substitution for a drug or other medically active treatment. 